\title{
Investigating Technologies in Decision based Internet of Things, Internet of Everythings and Cloud Computing for Smart City
}

\author{
Babur Hayat Malik ${ }^{1}$, Zunaira Zainab ${ }^{2}$, Husnain Mushtaq ${ }^{3}$, Amina Yousaf $^{4}$, Sohaib Latif ${ }^{5}$, Hafiz Zubair $^{6}$, Sayyam \\ Malik $^{7}$, Palwesha Sehar ${ }^{8}$ \\ Department of CS \& IT \\ The University of Lahore Gujrat Campus \\ Gujrat, Pakistan
}

\begin{abstract}
The idea of a Smart City features the need to upgrade quality, interconnection and execution of different urban administrations with the utilization of data and correspondence advances (ICT). Smart City advances cloudbased and Internet of Things (IoT) based administrations in which certifiable user interface utilize PDAs, sensors and RFIDs. Distributed computing and IoT are by and by two most essential ICT models that are forming the up and coming age of registering. Cloud computing speaks to the new technique for conveying equipment and programming assets to the clients, Internet of Things (IoT) is at present a standout amongst the most well-known ICT ideal models. In the meantime, the IoT idea imagines another age of gadgets (sensors, both virtual and physical) that are associated with the Internet and give diverse administrations to esteem included applications. Focus of this study attention on the integration of Cloud, IoT and IoE technologies for smart city services as well as a review has been made so that we can develop a better smart city that will utilize IoT, IoE in order to provide a better platform for smart city. This paper tends to the joined area of cloud computing, IoT and IoE for any smart city application organization.
\end{abstract}

Keywords-IoT; IOET; technologies; cloud computing; WSN

\section{INTRODUCTION}

In beam of the speedy rise of the public depth in the interior urban situations, substructures and administrations have been estimated to deliver the requirements of the society properly, there has been a marvelous enlargement of automated gadgets, for example, sensors, actuators, advanced mobile phones and brilliant apparatuses which constrain to substantial business goals of the Internet of Things (IoT), on the foundation that it is reasonable to be integrated all gadgets and make correspondences between them through the Internet [1].

The smart city is getting to be more brilliant than in the past because of the present development of computerized innovations [2]. Brilliant urban communities comprise of different sorts of electronic equipment connected by a few applications, for example, cameras in a checking framework.

\section{A. Internet of Things (IoT)}

The phrase "Web of Things" first showed up in 1999 when Ashton displayed a write about Radio Frequency Identification (RFID) to Procter and stake [3]. The option of involuntary information increase utilizing RFID and detecting improvement, in concert with the reliable enhancement on Wireless Sensor Networks (WSNs), Machine-to-Machine (M2M) structures, Artificial Intelligence (AI) [4] and semantic advances have empowered IoT to flourish. Cisco has estimated that 50 billion of things will be related with the Internet by 2020 , accountable to be 6.58 times more than the assessed total general public as shown in Fig. 1 .

Gadgets, PCs, and machines were at that point connected when Kevin Ashton authored the expression Internet of Things [5]. The suggestion pulled out up condensation for its competence to boundary the position apart - physical-first protests in advance unfitted for creating, transmitting and getting information unless stretched out or restricted. Installing sensors, control frameworks, and processors into these articles empowers even association over a multi-hub, open system of physical-first protests [6]. The term is as well apprehensively used to characterize related intricate first gadgets, for example, wearable contraptions that might be named Internet of Digital while submission an interchangeable worth from its physicalfirst partner formed into a smart connected originality [4]. The significance and use of the expression IoT will maintain on growing as new related advances expand, supplanting physicalfirst questions with luminous connected gadgets and develop cases to compose all new "Web of things" instructions. Belongings of IoT integrate connected autos, smart meters, and smart town communities, among others.

\section{B. Cloud Computing}

Presenting new modern services to residents in smart urban areas requires a massive application in gathering, putting away, and preparing information detected in the earth and created by natives themselves [5]. Cloud arrangements can enhance the nature of smart urban areas services. Offering financial assistance to hold on and to break down the information gathered, in this way to separate learning from the unsophisticated information obtained [7]. The expanding requirement for supporting collaboration between Internet of Things (IoT) and distributed computing frameworks has additionally encouraged the structure of the edge figuring model, which means to give handling and capacity limit as an expansion of accessible IoT gadgets, without the need to move information preparing to a focal datacenter as shown in Fig. 2. 


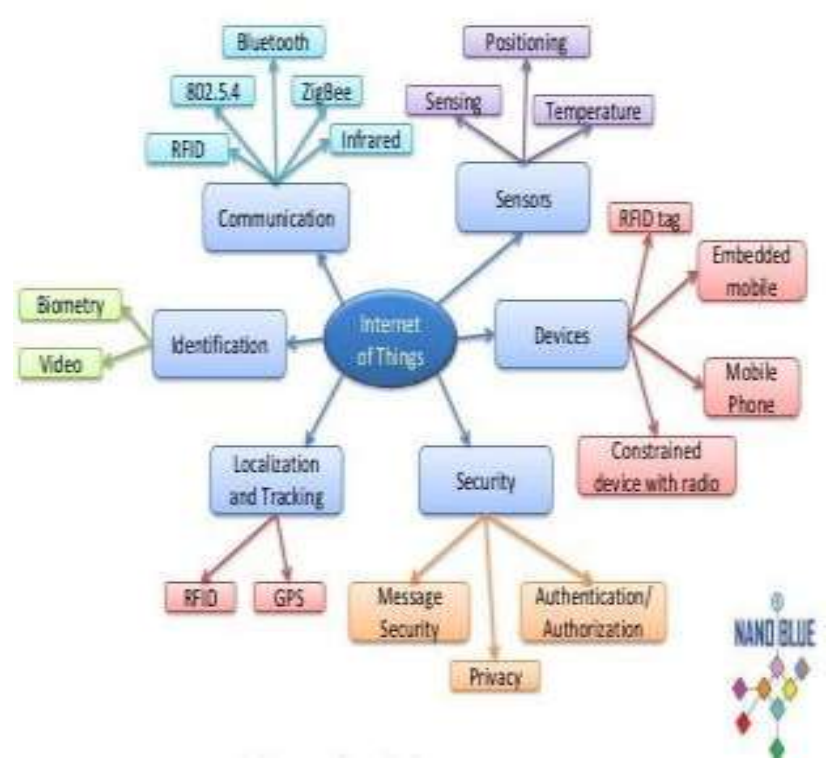

Fig. 1. (The IoT Connectivity)[3].

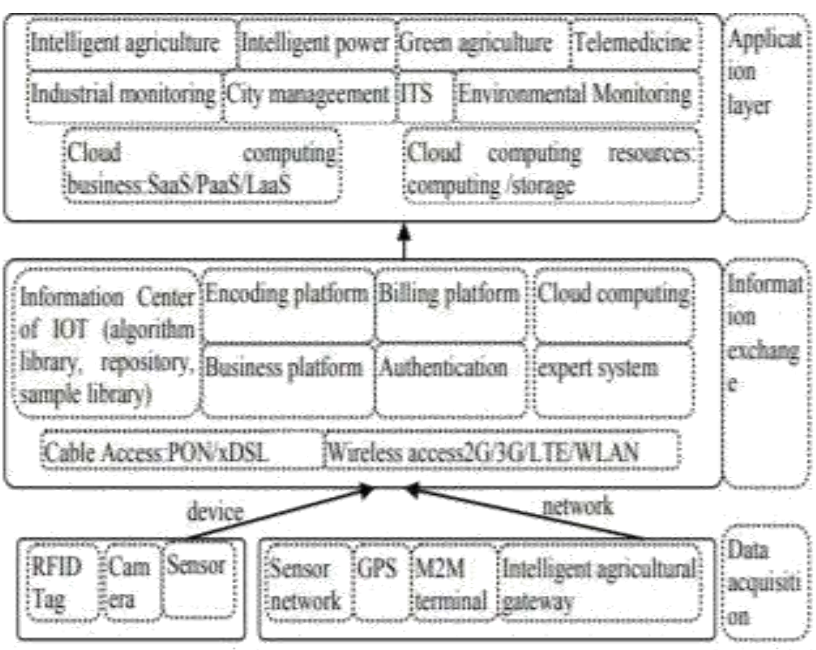

Fig. 2. (The Technical Architecture of IoT)[4].

The union of IoT, edge, and distributed computing requires an osmotic administration of services and micro services. Crosswise over various frameworks, where assets are progressively sorted out and relocated by the fundamentals of various foundations (e.g., load balancing, reliability, availability) and applications (e.g., detecting/activation capacities, setting mindfulness, district, Quality of Service (QoS). Osmotic registering empowers micro service and asset arrangement instruments, together with consistent movement of services that adjust their conduct as indicated by asset accessibility [2].

The face of that the idea of Internet of Everything rise as a characteristic improvement of the IoT development and is to a great extent connected with Cisco's strategies to start another advertising area, IoE includes the more extensive idea of availability from the viewpoint of present day network innovation utilize cases [8]. IoE involves four key components including a wide range of associations possible:
- People: Considered as end-hubs associated over the web to share data and exercises. Illustrations incorporate interpersonal organizations, wellbeing and wellness sensors, among others [6].

- Things: Physical sensors, gadgets, actuators and different things creating information or accommodating data from diverse sources. Illustrations integrate smart enclosed regulators and contraptions [8].

- Data: Raw information broke down and handled into valuable data to empower clever choices and control systems. Cases incorporate temperature logs changed over into a normal number of high-temperature hours every day to assess room cooling fundamentals [9].

- Processes: Leveraging network among information, things and individuals to include appreciate. Illustrations incorporate the utilization of smart wellness gadgets and informal communities to promote important social insurance offerings to planned clients [10].

IoE builds up a winding up to-end biological system of network including advancements, procedures and ideas utilized over all availability utilize cases [11]. Any further arrangements, for example, Internet of Humans, Internet of Digital, Industrial Internet of Things, correspondence innovations and the Internet itself - will in the long run constitute a subset of $\mathrm{IoE}$ if not considered in that capacity as of now.

\section{Smart Cities and IOT}

Smart citizens, smart energy, smart buildings, smart mobility, smart technology, smart healthcare, smart infrastructure, smart governance and education. The smart city is getting to be quicker witted than in the past because of the present extension of advanced trends. Smart urban communities comprise of different sorts of electronic device connected by a few applications, for example, cameras in an observing framework, sensors in a transportation framework, etc. Moreover, use of individual portable devices can be spread [11][12]. The smart city vision includes improving personal satisfaction by picking up information understanding from interconnected sensors, gadgets and individuals as shown in Fig. 3 [13] continual urban issues like security, dissipate administration and movement can be tended to by utilizing information to pick up efficiencies; however to do this the greater part of the information needs some place to go where it can be effectively gotten to and utilized by all partners, both private and governmental.

The two famous corporations IBM and Cisco used this term to give the concept of connected and computerized cities. The most important component of smart cities is the Information and Communication Technology chains. In the early 1990s, the debates on urban politics resulted in the progress of the concept of smartness. It is a model derived from another related concept, called "smart growth" which is a North American idea created by "New Urbanism" movement. The ideas of "smart growth" and "smartness" are strongly associated to the questions of economic environment and social justness. 


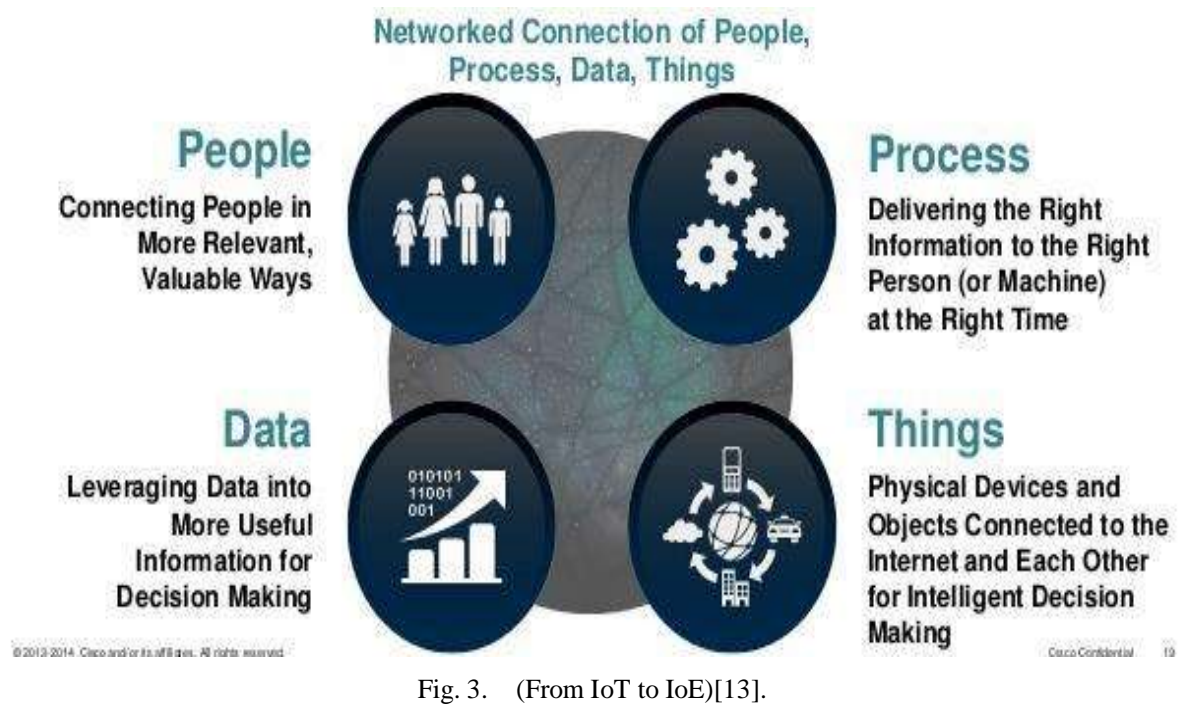

The idea of "smartness" is generally related to the concept of "smart growth" because it has a practical and realistic breadth. But, there is a huge difference between these two terms. A lot of cities use the term "smart" in their policymaking plan. The term "smart" in the policy making plans of cities means either expansion or contraction. The term "smart" is related to good practices while the "smartness policy" is linked to the special and sectorial areas [14].

There is a problem with the use of the term "smart cities", because it has different meanings. To some people it may mean the innovation in the technologies used in the cities and the betterment of living standard through ICT [15]. And to some people it means the use of technologies for better governance and for solving the social and environmental problems. Regardless of what it means to different people (whether it means industrial revolution to people or it means a better, technological and digital environment) a smart city must be a place where people can live easily, it must be socially comprehensive, and it must take care of the happiness, comfort, security, safety health and all other interests of its citizens.

\section{Cloud Support Data Management Infrastructure for Upcoming Smart Cities}

People living in the cities and enjoying the benefits of the technological advancements now wish to access their belongings and the facilities available in the cities all the time. To provide this facility to the people, cloud based services can be used. Because for providing the citizens with this kind of facilities, such a system is needed as can deal with the continuously growing city data, need for applications, processing capabilities for large data and application, and can store, share and transfer the data and information easily [6].

But, the processing of such a huge amount of data (big data) has the issues regarding cost and scalability [7]. By cost it mean not only the cost of processing, but also the cost that will incur on the transfer of such capacious data. One solution to this problem is to divide the process into four different levels i.e. replication, partitioning, caching and distributed control, just like Google and Azure are doing.
Whatever is left of the paper is sorted out as takes after. This area exposes the idea of IoT. Then it talks about purposed methodology and related work. At long last, it displays the conclusions and distinguishes open research difficulties to build up an IoT based disaster flexible correspondence arranges.

This paper discusses the latest research based on $\mathrm{IoE}$ technologies with the objective to classify them into a list of major requirement recommendations for building smart city systems. In this paper a review has been made so that can develop a better smart city that will utilize IoT, IoE in order to provide a platform that will be better for smart city [13]. Furthermore diverse technologies are compared to investigate technological framework in IoT, IoE and smart city in Table 1. Moreover it also gives a review on recent literature review so that can find out the optimized technology for smart city services. Study analyze the IoT and IoE in administrations however the IoE is more solid and effective for the time being a day's smart city chosen for the practical implementation of the smart city. Finally section vii, viii describe the comparison and conclusion. The expression or title "smart city" was created in the United States of America.

\section{LITERATURE REVIEW}

Smart city notion leave a noteworthy impression on the growth of nations. It enhances the strength of societies in order to take quick and effective decisions at the right time. Internet of things (IoT) appeared as a promising technology that link smart devices to address with social challenges and also assist big data analytics aimed at creation of smart cities worldwide.

The following section provides an overview that has been made of the work of recent researchers for smart cities by using IoT generated big data analysis.

In this article [14], recent literature was reviewed to explore the exceptional characteristics, components and features of the IoT based system. As it is known that the exertion of IoT infrastructure may facilitate number of opportunities, therefore utmost research motivations are defined at first and then expedient applications are bounded. This paper also explained 
the ways regular actions can be established and heightened by employing IoT. The challenges that emerge in implementation of IoT based system for smart cities were also comprehensively defined. Moreover, a mechanism is also provided to overwhelm few of the fundamental threats such as confidential rights of the residents.

This article [15] presents essentials of a smart city as far as definitions, principles and suggestions. To comprehend the significance of a smart city, all attributes and features are portrayed in a basic way. As smart city idea arise as an application area of IoT among different ideas that take advantage of Inter Computing Technology (ICT) in urban states such as innovative city, green city, ecological city, and intelligent city etc., smart city emerges inferable from its comprehensive perception. The literature discussed in this paper recognized that the acknowledgement of a smart city extremely builds upon information handling, pervasive approachability and platform dependent interoperability between devices.

The author in [16] presents a brief overview of the applications of IoT that are using in different departments of a smart city. The author proposed an IoT based model for smart street lighting system that will alter the intensity of light as indicated by needs or as per time. The projected framework takes advantage of sensors in order to identify human activities inside some characterized scope of light. If there is a human activity within that particular defined range then the lights gets turned on automatically, otherwise it stays off.

In this article [17] a systematic literature review (SLR) based on the previous literature and Indonesia's legal foundation, was directed to figure out what services are extremely required in improvement of a smart city development. The author, in this paper attempts to locate common and generic facilities for developing a smart city in Indonesia by conducting Synthesis research. This study too denotes a general guideline for Indonesian government to develop a smart city in Indonesia. The results of this study reveals that the smart city consists of different services, will proceed together so that to progress the value of the life of community as well as information technology will grow up as a tool to govern the realization of service endowment and the management will act as a supervisor intended for the employment of smart city.

The author in [18] gave a comprehensive review on an urban IoT, named "Padova Smart City" that has been recognized in the city of padova, made conceivable by the meeting up of various gatherings, for example district of padova supported the project. The major aim of this project was to indorse early open data implementation and ICT solutions for public administration. The target application comprises of a framework that would accumulate the conservational data and then monitor the community street lighting through the use of wireless nodes. The application armed with different types of sensors have been set on the street light poles and is associated with the internet through an access element which is utilized for sending data.

The author in [19] discuss about the behavior of smart city in combined system of urban infrastructure management. An
IoT centered System designed for smart cities is presented, called "SIGINURB". The idea behind this system is to construct a scope of requests that offer novel facilities to students, employees, public and business administration in the University of São Paulo, educating their personal satisfaction. "SIGINURB" is an infrastructure whose main aim is to enhance the ease and value of life of the people of university São Paulo. This framework at first, coordinates with automatic systems to offer tangible verdict that encompass deliberate environment. This idea has been coordinating to redesign the ancient procedures like open lighting, power supply, dispensing water and observing the climate conditions. Furthermore, a number of various applications have also been identified and demonstrate the framework conducted in each circumstance.

The author in [20] investigate about the potential for Artificial intelligence (AI) that can handle IoT and big data and further cities using ML strategies to find the association among weather-based situations and short- cycling journeys in London. For a successful personalized service, it is important to appreciate the behavior of users for particular conditions and must have the capacity to accommodate services that well fit their needs. Four well known ML classification algorithms are used that were operated on the data taken from six datasets. Each classification algorithm were reliable and produce high accuracy results. The result of this study shows that KNN algorithms remained inappropriate for small computations. The reason behind this is that a lot of time is needed to properly educate the algorithm however decision tree algorithms was proved to be appropriate for those applications where accuracy was important. Moreover, the results indicates that a arrangement of ML, IoT and big data techniques suggest excessive prospective to creators of smart city knowledge and facilities.

The author in [21] considers application and prospects of exhausting the Cloud of Things (CoT) that supports fog computing in order to provide smart city services. All the concerns for employing such services, issues and challenges are identified in order to realize enhanced consumption of CoT in improving smart city services. Headed for exact and competent decision making meant for smart city services, CoT offers powerful cloud platforms to run, implement and back together online and offline smart city service optimization. By using CoT, Fog computing and other useful technologies, integrating services provide a lot of prospective to maximize services that may uplift the effectiveness and control of smart city services. However, the issues related to CoT architecture, strategy, security, confidentiality and suitable optimization techniques needs to be fixed in order to attain good results.

The author in [22] presents the implementation of IoT techniques for management of resources. To raise the efficiency of energy management of cities, different software solutions have been proposed. Energy management also involves immersive monitoring and estimation of energy information. Two distributed platforms are considered that are established to expand the energy management in smart cities.

The author in [23] tends to address the synchronized field of cloud computing and IoT for the deployment of any smart 
city application. The characteristics of cloud platform that are mandatory for the development of any smart city and also the authentication of platform's capacity personalized to IoT functionalities using cloud middleware have been discussed. Dubai as a smart city is also talked about with nearly application - based situations launched by smart city enterprises. A study on the utilization of smart city cloud interoperability and connectivity has also been reviewed. This paper also proposed an IoT based framework for healthcare management systems.

The overall purpose of this paper [24] is to comprehend the ways through which smart cities may vary in context of its meaning, intensions and offerings. Several interpretations relating to the construction of smart cities have been discussed and thus a 3RC framework have been proposed that consists of Preventive, Contemplative, Rationalistic and critical schools, to fundamentally investigate different stages in the advancement of the field. This article [24] proposed and developed a smart city system, centered on IoT expending big data analytic techniques with sensor deployment and IoT based smart systems. Various smart systems generated data are acquired to provide real time decision making for smart cities .Using Hadoop network in real situation, complete architectural and execution model has been proposed.

The Hadoop network is all set to practice big data that would be produced via totally smart systems established in the city. Implementation phase comprises of different stages that begins after statistics creation and gathering, aggregation, categorization, sorting, preprocessing computing and finishes at decision making. The effectiveness in big data dispensation is accomplished by consuming catalyst over Hadoop. This method remains virtually executed and tested on real data. The estimation result shows that projected system is highly accessible and competent Section IV gives a comprehensive overview of methodologies regarding IOT and IOE as given below in Table 1.

\section{TeChnOlogies ChOOSEN FOR THE PRACTICAL IMPLEMENTATION OF THE SMART CITY CONCEPT}

The population is growing rapidly in the urban areas. One of the reason why people prefer to live in urban areas or the reason of the urbanization is the availability and provision of all the basic facilities that are needed for today's life. On the other hand, digital devices, smart phones, sensors and cameras have been growing and improving very quickly over the past few years. So, the growth of population in the cities and the growth of digital devices gave birth to a unique idea of building smart cities. There was a huge potential for the business, if the citizens were provided with the facilities using all these devices and technologies. Also, the Internet has now made it possible to make all the devices part of a network. Gathering information, for example, of a public transport (e.g. current location, consumption of the parking places, traffic blockage, and traffic jams, etc.), and the correlated data (e.g. weather conditions, air contamination and noise pollution, toxic waste in the water, smog and energy consumption etc.) has now become very easy as shown in Fig. 4 [21]. But, careful selection of the technologies, that are going to be used in a smart city, is very important.

The power of IoT is in the self-configuring devices linked globally [23]. One can understand the concept of IoT by considering the IoT a single entity, which is detached for the most part, with less storage and processing potential. IoT determines to provide high dependability, functioning and safety of the smart cities and their infrastructure as well [22].

Internet of thing is everywhere now. Fig. 1 is directing towards the recent development facet of IOT that based on technological and systematic levels in IOT to IOET. Sensing technology is the valuable technology. The IoT consists of three layers. The following picture clears the concept:

\section{A. Perception Layer}

This layer contains internet enabled devices which can provide the facilities of communication and exchange/transfer of data. The examples are Radio Frequency Identification Devices (RFID), Global Positioning System (GPS), sensors and cameras etc.

\section{B. Network Layer}

This layer in the IoT systems is formed with the blend of short and long-range communication technologies such as Bluetooth, ZigBee, Wi-Fi, 2G, 3G, 4G and Power Line Communication (PLC), etc.

\section{Application Layer}

This layer receives the information and processes it. Dividing the IoT in the layers helps us make effective power distribution and management plans for the smart cities.

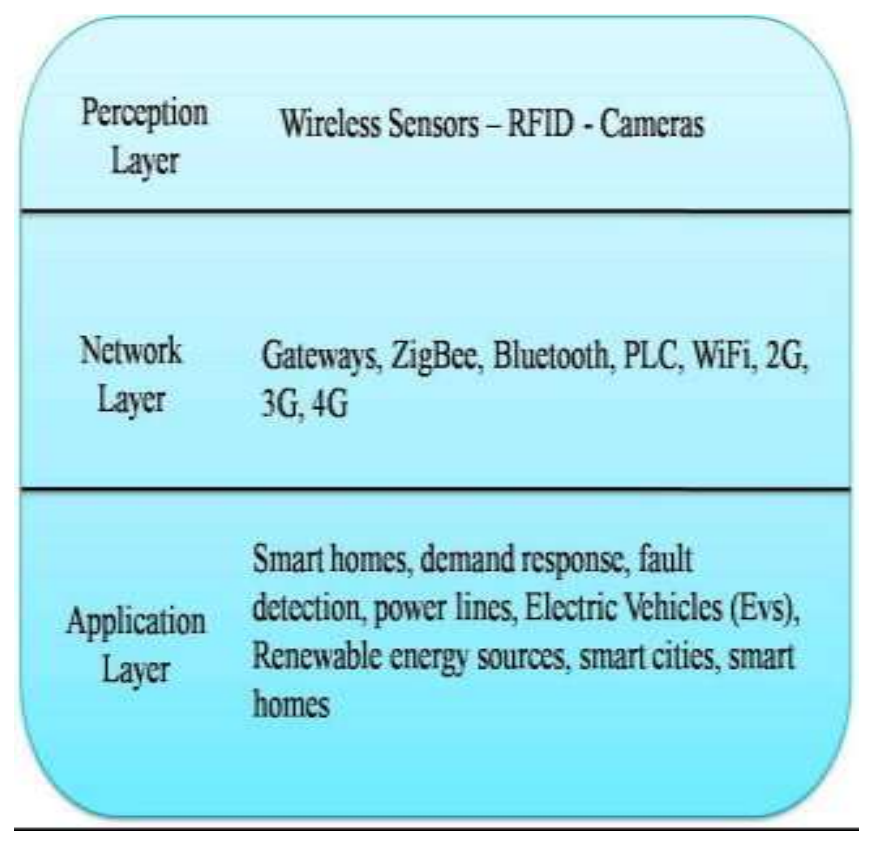

Fig. 4. (Layer Architecture of IoT) [21]. 


\section{COMPARISON}

TABLE I. OVERVIEW OF METHODOLOGIES REGARDING IOT AND IOE

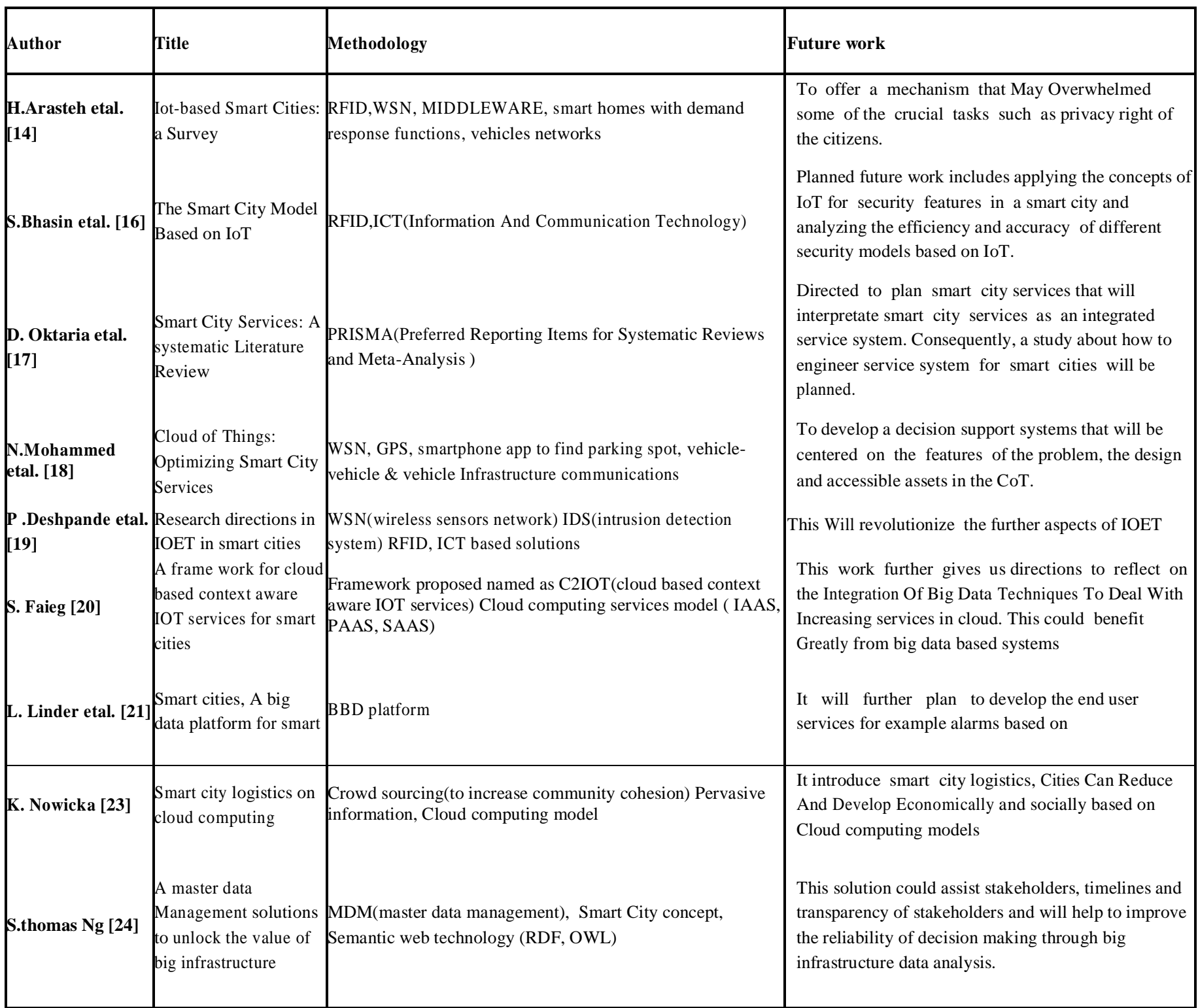

\section{IOT TECHNOLOGIES FOR SMART CITIES}

The Internet of Things is thought of as a communication network (broadband) that uses standard communication models [23]. The Internet is used as a junction or union. The practical implementation of the IoT model is based on the wireless communication. Interconnection among different objects can be made a reality using even a low-power standard communication system [21]. Following are some networks that have been defined on the basis of location and the size of the area they can provide communication facility to:

\section{A. Home Area Network}

It uses short-range communication standards e.g. Wi-Fi to connect all the supervising and organizing components in a home.

\section{B. Home Area Network}

It is used to make communication among customer and the resource distributors which require much greater area coverage than the Home Area Network model.

\section{Home Area Network}

It provides communication between the client and substation [16]

In the following section, a small explanation of the IoT related technologies is given:

- Radio Frequency Identification (RFID)- Including the readers also the tags have an important role in the skeleton of IoT): Implementing the technologies on each related object, getting their automatic identification, and assigning a single digit identity to 
any such object will be possible [12]. Some of the services that RFID provides are track and locate objects, healthcare applications, parking lots, and asset management etc.

- Near Field Communication (NFC) - It is useful for two way connections in small areas. If this technology is used in smart phones, then the smart phones will also be able to be used in the smart cities. One example of the use of NFC in smart phones can be the use of smart phones as our wallets. The smart phone can then be used as cards in our possession (bank cards, identity cards, transportation cards and access cards etc.). NFC is a two way communication standard, therefore it can also be used for transferring data among devices [11]. Also it can help use change the status of the devices e.g. one can turn on Wi-Fi with the help of this standard.

- Low Rate Wireless Personal Area Network (LWPAN) (IEEE 802.15.4) - It is a radio technology that covers small areas (10 to $15 \mathrm{~km}$ ). This range makes it suitable for smart cities. It consumes less energy and battery life may be up to 10 years [15]. This standard has been defined as the cost-effective and short area communication provider for WSN networks. In addition to the protocols of the upper layer for instance ZigBee and Wi-Fi and 6LoWPAN [2], it includes the two layers of protocols that are at the bottom including physical and medium access levels.

- ZigBee- A cost effective and requiring less power wireless communication technology (IEEE 802.15.4). It is very useful for Wireless Personal Area Networks (WPAN). For example computerized homes, medical devices control and other requiring a low bandwidth, cost and power [6]. Some of the application may be the light switches (wireless), electrical meters, and systems for managing the road traffic. Communication among millions of devices in a smart city is possible using ZigBee is easily possible. It utilizes the IPv6 addressing and some additional equipment is needed for its implementation such as ZigBee end devices, a coordinator and router.

- 6LoWPAN- This has been mentioned to adapt to the IPv6 addressing, because the previous version of addressing which is IPv4 is slowly being overridden by the IPv6 because IPv4 doesn't have addresses that have not been used and it is also not capable of assigning addresses individually to billions of devices, which is the basic requirement of IoT networks. The IPv6 provides 128-bits addresses and has enough address space to support the devices in a smart city environment. But, it creates another problem that some of the old devices can't support IPv6. So, the solution has been found in the form of 6LoWPAN that is a compressed format of IPv6 .

- Wireless Sensor Networks (WSNs) - These Sensor Networks make miscellaneous data available and can be useful for various fields such as healthcare and governmental and environmental services etc. [2]. It can also be combined with the RFIDs to gain many objectives such as obtaining information about the current location of different men and women and other things, their heartbeat and body-temperature, for instance. A wireless sensor network is mainly made with the help of sensor nodes that work wirelessly. These nodes have electro-magnetic waves (radio) interference, converter (analog-digital), a number of sensors, storage and to make all the components function properly power supply is required [5]. The following diagram gives a clear understanding of the details given in this paragraph. A wireless sensor node is usually very small and can be applied in any environment. The only limitation of a wireless sensor node is the dependence on the power supply. Because the battery life is limited so there must be found other ways to supply the power such as solar energy can also be used for this purpose

- Dash7- A very good standard for Wireless Sensor Networks, provides long range and support low power applications e.g. smart buildings and logistics etc. It is an ideal networking standard for HANs. It operates at 433-MHZ and provides better walls penetration than 2.4-GHZ [9]. Dash is a medium range standard and is known as kilometer-range protocol. It seems very attractive for military applications, such as building substations. Some other application examples may be unsafe material monitoring, manufacturing and go down betterment and the development of smart meters etc as shown in Fig. 5.

- 3G and Long Term Evolution (LTE) - Technologies for communicating wirelessly using mobile phones and the devices for data storage (data terminals). These technologies are now available everywhere in the world. Because, mobile phones have now become the necessity of each and every individual these days. These technologies are for long range communication and broadband services. These are used for Wide Area Networks which require long area coverage. However, there are a few problems in the practical use of these services which must be addressed. Main issues are the high data costs from the providers and the limitations in the devices from the manufacture to not to support these technologies.

- Addressing- The internet provides the facility of interconnection among billions of devices. So the addressing schemes must be made better, because the IoT aims at connecting almost all the physical object on yhe planet to the networks [8]. For smart cities, currently IPv6 is a suitable addressing scheme, which supports billions of devices and has space for more

- Middleware- Because of the difference in the nature of devices, limited storage capacity, limited processing resources, and countless applications of different types, the middleware has a very vital role in the implementation of IoT and smart city concepts for connecting the things at the application layer. The main aim of this middleware is to combine the working 
mechanism and capabilities of communicating of all the contributing devices.

- Smart Cities Platforms and Standards- The combination of physical devices and the overall IT skeleton makes a very unique communication among devices for smart cities [3]. This adds new abilities to the networks and runs the communication platforms of smart cities [15]. The requirement of supporting heterogeneous applications and devices can easily be fulfilled with such kind of platforms. These platforms also support the implementation of IoT and sensor networks. One example of such plat forms is widely used open MTC [19]. It provides a middleware platform that supports the machine-machine communication and the implementation of smart cities [2].

\section{COCLUSION}

This paper tends to the vision that the private structures would move themselves toward modern unit that would be a development of the submissive areas. This paper has proposed a rundown of prerequisites for building smart city. The seven noteworthy innovations purposed in this paper. Meeting these prerequisites does not equivalent to a framework that everyone will utilize yet it gives a typical stage to building a smart city. The primary commitment of this paper is the IOET allencompassing structure which fuses diverse ideas from IOT which are purposed in the literature. There is an absence of a binding together stage that would change these individual and separate applications into a solitary foundation .The arrangement of this issue is the IOET which cover every one of these people applications into a solitary stage .

\section{REFERENCES}

[1] C.Yang, etal "Utilizing Cloud computing to address big geospatial data challenges", Computers, Environment and Urban Systems, vol.4, no.61, pp.120-128, 2017

[2] L.Zhuhadar, et al "The next wave of innovation Review of smart cities intelligent operation systems", Computers in Human Behavior, vol.4, no.66, pp.273-283, 2017.

[3] Anthopoulos, L., Janssen, M., \& Weerak kody," Smart service Portfolios: Do the cities follow standards?" "In Proceedings of the 25th International conference companion on world wideWeb: "International world wide web conferences steering committee",

[4] S.Talari, P. Siano, et al "A Review of Smart Cities Based on the Internet of Things Concept", "journal of Energies”, vol.4, no.10, pp.421, 2017.
[5] J.Jina, M.Palaniswamib, et al "An Information Framework of Creating a Smart City through Internet of Things", vol.4, 2017.

[6] G.SUCIU, et al "cloud computing and internet of things for smart city deployments", "Software Workbench for Interactive, Time Critical and Highly self-adaptive Cloud applications", 2014.

[7] Biljana L. Risteska Stojkoska "A review of Internet of Things for smart home: Challenges and solutions", Journal of Cleaner Production, 2016.

[8] [Online]. Available: Sudeep Tanwar, Sudhanshu Tyagi, Sachin Kumar, Lecture Notes in Networks and Systems, vol. 19, pp. 23, 2018, ISSN 2367-3370, ISBN 978-981-10-5522-5. Accessed: March. 16, 2018.

[9] [Online]. Available: Syed Muhammad Waqas Shah, Maruf Pasha, "IoTBased Smart Health Unit", Journal of Software, vol. 12, pp. 45, 2017, ISSN 1796217X. Accessed: March 13, 2018.

[10] Hui, T. K. L., Sherratt," Major requirements for building Smart Homes in Smart Cities based on Internet of Things technologies", 2017.

[11] [Online].Available:https://publications.computer.org/cloudcomputing/2017/05/17/convergence-of-iot-edge-and-cloud-computingin-smart-cities-call-for-papers/ Accessed: March 24, 2018.

[12] H.Aratesh etal , "IoT- Based Smart Cities: a Survey", IEEE, 2016

[13] B. Silva, M. Khan and K. Han, "Towards sustainable smart cities: A review of trends, architectures, components, and open challenges in smart cities", Sustainable Cities and Society, vol. 38, pp. 697-713, 2018.

[14] S. Bhasin, T. Choudhury, S. C. Gupta, P. Kumar, "Smart City Implementation Model Based on Iot," IEEE, 2017

[15] D. Oktaria, Suhardi, N.B.Kurniawan, "Smart City Services: A Systematic Literature Review", in International Conference on Information Technology Systems and Innovations, IEEE, 2017.

[16] A. Zanella, N. Bui, A. Castellani, L. Vangelista and M. Zorzi, "Internet of Things for Smart Cities", IEEE Internet of Things Journal, vol. 1, no. 1, pp. 22-32, 2014.

[17] L.B.Campos etal., "Towards an IoT - Based System for smart City", "IEEE International Symposium on Consumer Electronics", 2016.

[18] J. Chin, V. Callaghan \& I.Lam , "Understanding and Personalizing smart City Services Using Machine Learning, The Internet of Things and Big Data ", IEEE, 2017.

[19] N.Mohammad, S. L. Molnar \& J. A. Jaroodi, "Cloud of Things: Optimizing Smart City Services", IEEE, 2017.

[20] E.Patti \& A. Acquaviva, "IoT platform for Smart Cities: requirements and implementation case studies", 14 November 2016.

[21] M. J. Kaur \& P.Maheshwari, "Building Smart Cities Applications Using IoT and Cloud - Based Architectures", IEEE, 2016.

[22] R. Kummitha and N. Crutzen, "How do we understand smart cities? An evolutionary perspective", Cities, vol. 67, pp. 43-52, 2017

[23] M.M.Rathor, A.Ahmed \& A. Paul, "IoT - Based Smart city Development Using Big Data Analytical Approach", computer networks, vol.101, pp. 63-80 2 